\title{
AN INVENTORY SYSTEM FOR PERISHABLE COMMODITIES WITH RANDOM LIFETIME
}

\author{
DAVID PERRY* Haifa University
}

\begin{abstract}
In this study we assume an inventory system for perishable commodities in which the lifetimes of the items stored are i.i.d. random variable with finite mean.

We utilize the analogy between this inventory system and a queueing system with impatient customers, to study the process of the lost demand, the death of the unused items and the number of items in the system.
\end{abstract}

VIRTUAL WAITING TIME; VIRTUAL LOST DEMAND TIME; $M / G / 1$ QUEUEING SYSTEM; BUSY PERIOD; KEY RENEWAL THEOREM

Two recent papers [1] and [2] treated an inventory system in which the lifetimes of the items stored are fixed and finite. In this study we assume that the lifetimes of the items stored are i.i.d. random variables with finite mean; note that in this case it could happen that an older item dies before a younger one. For a FIFO issuing policy we utilize the analogy between our inventory system and a queueing system with impatient customers to study the processes of the lost demand, the death of the unused items and the number of items in the system.

The analysis of this model is dual to that of the model we considered in Section 5 of [2], in the sense that both of them are analogous to the same queueing model (where $\lambda$ and $\mu$ change roles with each other).

We assume that the arrival of items into the system is a Poisson process with rate $\lambda$, and the arrival of demands is a renewal process with inter-renewal distribution $B$ and mean $1 / \mu$. When a demand occurs and there are items in the system the demand is satisfied immediately by the oldest item, otherwise it leaves the system unsatisfied. An item which was not taken by a demand during its lifetime (which is assumed to be a random variable having distribution $H$ with finite mean) leaves the system.

Define a process $\tilde{W}=\left\{\tilde{W}_{t}: t \geqq 0\right\}$ such that $\tilde{W}_{t}$ is the time of the next unsatisfied demand from $t$, if the arrival process of items is stopped at time $t$. We call this process the virtual lost demand time.

It is easy to see that the time between lost demands is a delayed renewal process. Let the origin be a time of a lost demand and $D$ be the time between lost demands. Then, using the analogy to the queueing system (see also Section 5 of [2]), $\tilde{W}_{t} .1_{\{0 \leq t<D\}}$ is stochastically equal to the virtual waiting time in an $M / G / 1$ queueing system with arrival rate $\lambda$, and service time distribution $B$, in which customers join the system if they have to wait for $x$ units of time with probability $1-H(x)$, and leave with probability $H(x)$.

Received 6 February 1984; revision received 9 October 1984.

* Postal address: Department of Statistics, University of Haifa, Mount Carmel, Haifa 31 999, Israel. 


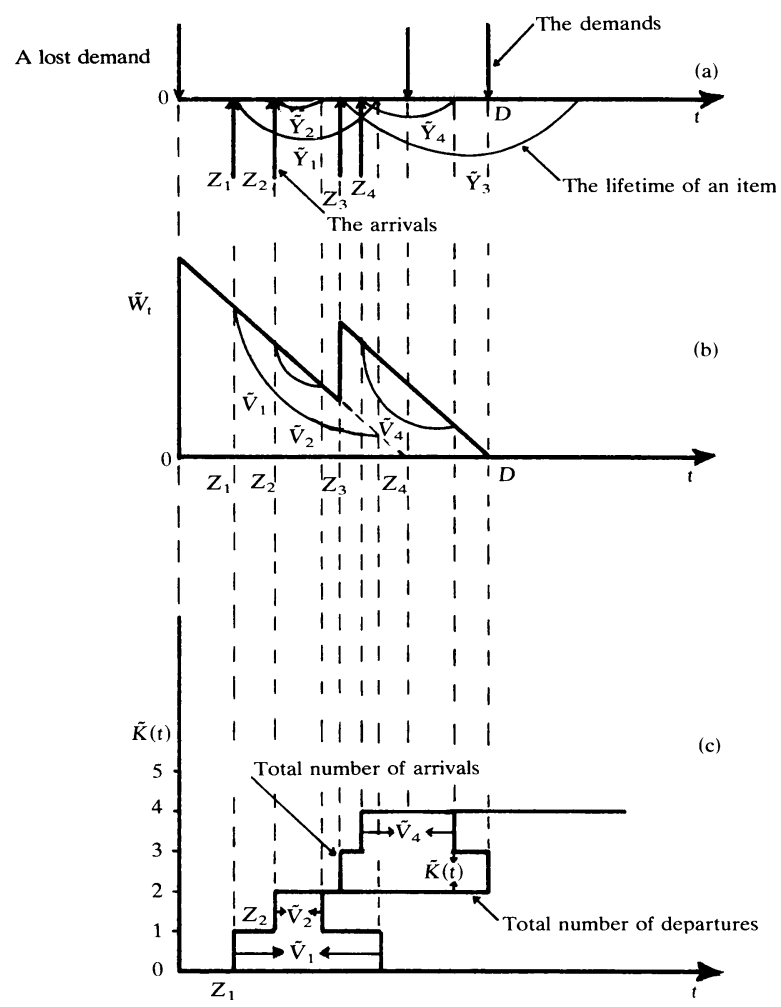

Figure 1(a), (b) and (c). The arrival, demand, virtual lost demand time, and number of items processes

Remark. The complete analogy between the queueing and the inventory systems is as follows. The time that a customer is ready to wait against the lifetime of an item, the arrival of customers against the arrival of items, and the service time against the times between successive demands. (For a typical realization see Figure 1(a) and (b).) Hence, the time between successive lost demands is stochastically equal to the busy period $-R$ in the above-mentioned $M / G / 1$ queueing system.

From the point of view of the distribution of $R$, it does not matter if the customer who arrives into the system leaves it immediately, when the virtual waiting time is greater than the time he is ready to wait, or whether he joins the system and leaves it after the time he is ready to wait has elapsed. We thus consider a queueing system in which every customer always joins it and focus on the analogy between $K(t)$-the number of customers in the above-mentioned queueing system at time $t$, and $\tilde{K}(t)$ - the number of items in the inventory system, at that time. There is, however, a difference between the two processes due to the fact that in the queueing model, the beginning of a busy cycle is the time of the first arrival to an empty system, while in the inventory model the beginning of a renewal cycle is the time of a lost demand. Let $N$ be the number of customers who arrived during a busy cycle in the queueing model, and let $\tilde{N}$ be the number of items in the inventory system which arrived between two lost demands; the random variable $\tilde{N}$ has the same distribution as $N-1$. There is another difference between the queueing system and the inventory system. The time that a 
customer spends in the queueing system is $Y_{n} .1_{\left\{W_{n}>Y_{n}\right\}}+\left(W_{n}+S_{n}\right) .1_{\left\{W_{n} \leqq Y_{n}\right\}}$ where $S_{n}$ is the service time of the $n$th customer, $Y_{n}$ is the time that the $n$th customer is ready to wait, and $W_{n}$ is the actual waiting time. The time that an item spends in the inventory system is $\tilde{V}_{n}=\min \left[\tilde{W}_{n}, \tilde{Y}_{n}\right]$ where $\tilde{Y}_{n}$ is the maximal lifetime of the $n$th item, and $\tilde{W}_{n}$ is the actual lost demand time. With the above in mind, we now obtain the limiting expectation of the number of items in the system. The following theorem is analogous to that of Theorem 3.6.2 in [3] (p. 83), but due to the principle difference between the inventory and the queueing systems, we give the proof in full.

Theorem.

$$
E \tilde{K}=\lambda \int_{0}^{\infty}\left(1-F_{\tilde{\mathbf{W}}}(x)\right)(1-H(x)) d x
$$

where $H$ is the distribution of $\tilde{Y}_{n}, E \tilde{K}=\lim _{t \rightarrow \infty} E \tilde{K}(t)$, and $F_{\tilde{W}}(x)=\lim _{t \rightarrow \infty} P\left(\tilde{W}_{t} \leqq x\right)$ is given in Theorem 5.11 of [2] where $\lambda$ and $\mu$ change roles with each other.

Proof. At a time of lost demand, the inventory system is empty; thus $\tilde{K}(t)$ is a regenerative process, with respect to the lost demand stopping times. It therefore follows from the key renewal theorem (see also [1] and [2]) that $E \tilde{K}=$ $(1 / E D) E \int_{0}^{\mathrm{D}} \tilde{K}(t) d t$. Imagine that a reward at rate $\tilde{K}(t)$ is earned at time $t$. Then, $E \int_{0}^{\mathrm{D}} \tilde{K}(t) d t=E \sum_{i=1}^{N} \tilde{V}_{i}$, since $\tilde{V}_{j}$ represents the reward on day $j$ (see Figure 1(c); both $\tilde{V}_{1}+\tilde{V}_{2}+\cdots+\tilde{V}_{\tilde{N}}$ and $\int_{0}^{\mathrm{D}} \tilde{K}(t) d t$ represent the area of the enclosed curve). Now, $D$ and $R$ have the same distribution, so that $E D=E C-E X$ where $C$ is the busy cycle, $X$ is the interarrival time and $R$ is the busy period in the queueing system. Therefore

$$
E \tilde{K}=\frac{E \sum_{i=1}^{\tilde{N}} \tilde{V}_{i}}{\frac{1}{\lambda} E N-\frac{1}{\lambda}}=\lambda E \tilde{V}=\lim _{n \rightarrow \infty} E\left(\tilde{W}_{n}, \tilde{Y}_{n}\right) .
$$

Since the arrival of items of Poisson process, $\tilde{W}_{n}$ has the same limiting distribution as $\tilde{W}_{t}$, i.e. $\lim _{n \rightarrow \infty} P\left(\tilde{W}_{n} \leqq x\right)=\lim _{t \rightarrow \infty} P\left(\tilde{W}_{t} \leqq x\right)=P(\tilde{W} \leqq x)$.

Now, $\left\{\tilde{Y}_{n}\right\}$ is a sequence of i.i.d. random variables having distribution $H$, independent of $\tilde{W}$, so that $E \tilde{V}=\int_{0}^{\infty} P(\tilde{W}>x) P(\tilde{Y}>x) d x$, and the theorem is proven.

In the special case when $\tilde{Y}_{n} \sim \exp (\eta)$ and $\tilde{S}_{n} \sim \exp (\mu)$, we can obtain the limiting distribution of $\tilde{K}(t)$ itself. In that case $\tilde{K}(t)$ is a birth and death process whose steady-state probabilities $P_{\tilde{K}}$ satisfy the set of equations $\lambda P_{\tilde{K}}=[\mu+(\tilde{K}+1) \eta] P_{\tilde{K}+1}$, $\tilde{K}=0,1,2, \cdots$. We can also obtain the Laplace transform of $D$ as we did in the dual system in Section 5 of [2].

\section{References}

[1] Kaspi, H. and Perry, D. (1983) Inventory systems of perishable commodities. Adv. Appl. Prob. 15, 674-685.

[2] Kaspi, H. AND Perry, D. (1984) Inventory systems for perishable commodities with renewal input and Poisson output. Adv. Appl. Prob. 16, 402-421.

[3] Ross, S. M. (1983) Stochastic Processes. Wiley, New York. 